\title{
A test of the CaR-FA-X mechanisms and depression in adolescents
}

Article

Accepted Version

Fisk, J., Ellis, J. A. and Reynolds, S. A. (2019) A test of the CaR-FA-X mechanisms and depression in adolescents.

Memory, 27 (4). pp. 455-464. ISSN 0965-8211 doi:

https://doi.org/10.1080/09658211.2018.1518457 Available at https://centaur.reading.ac.uk/78805/

It is advisable to refer to the publisher's version if you intend to cite from the work. See Guidance on citing.

To link to this article DOI: http://dx.doi.org/10.1080/09658211.2018.1518457

Publisher: Psychology Press

All outputs in CentAUR are protected by Intellectual Property Rights law, including copyright law. Copyright and IPR is retained by the creators or other copyright holders. Terms and conditions for use of this material are defined in the End User Agreement.

\section{www.reading.ac.uk/centaur}

\section{CentAUR}

Central Archive at the University of Reading

Reading's research outputs online 


\section{A Test of the CaR-FA-X Mechanisms and Depression in Adolescents}

Jeni Fisk, Judi A. Ellis, and Shirley A Reynolds

School of Psychology \& Clinical Language Sciences, University of Reading

Corresponding author:

Jeni Fisk

School of Psychology and Clinical Language Sciences

University of Reading, Reading, RG6 6AL, UK.

Telephone: +44 (0)1183786573

Email: j.fisk@pgr.reading.ac.uk

Word count (including manuscript, supplementary tables and all references): 8,902 


\begin{abstract}
People who have depression have difficulty recalling specific autobiographical information (Sumner, 2011). This is called overgeneral autobiographical memory (OGM) and is associated with the development and persistence of depression. Williams and colleagues (2007) proposed that OGM is maintained by three mechanisms: capture and rumination (CaR), functional avoidance (FA), and impaired executive control (X), and integrated these into the CaR-FA-X model. The aim of this study was to assess OGM and test the CaR-FA-X model in adolescents with low mood. We recruited 29 young people aged 12-17 with elevated symptoms of depression and 29 with minimal symptoms of depression, matched for gender and age. After controlling for IQ, adolescents with elevated depression retrieved fewer specific memories, ruminated more, and had poorer working memory and verbal fluency than adolescents with minimal depression. The groups did not differ on measures of inhibition or functional avoidance. The CaR-FA-X model was therefore partially supported. These results confirm that there is a relationship between low mood and OGM in young people and that OGM may arise as consequence of impaired working memory and verbal fluency and cognitive interference due to rumination.
\end{abstract}

Key words: overgeneral memory, adolescents, CaRFAX, depression 


\section{A Test of the CaR-FA-X Mechanisms and Depression in Adolescents}

Depression is a common mental health problem across the life span (Angold, Costello, Worthman, 1998) and the incidence of depression appears to peak during adolescence (Green, McGinnity, Meltzer, Ford \& Goodman, 2005). Depression during this period of life is associated with many adverse impacts e.g. impaired social functioning, (Groeben, Perren, Stadelmann \& von Klitzberg 2011) and poor academic achievement (Fergusson, Boden \& Horwood, 2007; Verboom, Sijtsema, Verhulst, Penninx, \& Ormel, 2014). Further, experiencing depression in adolescence is related to long term health difficulties including repeated episodes of depression (Lewinsohn, Allen, Seeley \& Gotlib, 1999) and future development of mental health problems (Boden, Fergusson, \& Horwood, 2007). For these reasons, it is important to establish if and how depression in adolescence is distinctive from depression that occurs later in life.

Across the lifespan, depression is associated with impaired recall of significant personal memories (Williams et al., 2007). Personal, or 'autobiographical' memories are important to our well-being and functioning; they help develop our sense of identity, identify goals and make decisions (Conway \& Pleydell-Pearce 2000). In addition, it has been proposed that the ability to retrieve specific autobiographical memories guides present and future thoughts and behaviours, and helps us regulate emotions (Raes, Hermans, de Decker, Eelen \& Williams 2003). Because adolescence is a critical period for developing our sense of self, to make plans for the future, and to pursue goals relating to these plans, we expect that disruptions in retrieving autobiographical memories during this period may have particularly salient impacts on current and future well-being.

The inability to retrieve specific memories is termed overgeneral memory (OGM: Williams and Broadbent, 1986). Overgeneral memories can be split further into 'extended' memories that last longer than a day (e.g. "on my holiday in Spain") and 'categoric' 
memories, which are a group of repeated events (e.g. "parties with friends"). The association between OGM and depression in adults has been replicated many times (Sumner, 2012) and there is a standard method of assessing OGM through the use of the Autobiographical memory test (AMT) (Williams \& Broadbent 1986). Participants are asked to retrieve a specific memory in response to a cue word (Williams et al., 2007) e.g. 'happy'. 'In adults, OGM predicts the course of depression independent of current depression and is associated with longer recovery time (Brittlebank, Scott, Williams \& Ferrier, 1993; Herman et al., 2008) Individuals in remission of depression also retrieve more overgeneral memories than neverdepressed controls (Williams et al., 2007). OGM is therefore considered an important cognitive marker in depression and has led clinical researchers to speculate about potential interventions to improve memory specificity (Dalgleish \& Werner- Seidler 2014).

OGM is also observed in depressed young people (e.g. Park, Goodyer \& Teasdale, 2002; Swales, Williams, \& Wood, 2001) and may increase vulnerability for the development and reoccurrence of depression (Kuyken \& Dalgleish 2011; Park et al., 2002). For example, in girls with a family history of depression, OGM predicted the onset of depressive symptoms one year later (Rawal \& Rice, 2012a). Similarly, Sumner et al., (2011) found that in teenagers, aged 16-18 years with a history of depression, reduced memory specificity predicted the reappearance of symptoms following an interpersonal stressor over a period of 16 months.

The self-memory model (Conway and Playdell-Pearce 2000) describes the processes involved in the construction and recall of an autobiographical memory. The model describes an interaction between a knowledge base (information stored in memory that is built over a lifetime) and the "working self" to influence what is encoded and retrieved. The knowledge base is organised in a hierarchy. The broadest (top) level of the hierarchy consists of general knowledge about a time in an individual's life (e.g., when I was at secondary school). 
Subsequent levels include more detailed events (e.g., attending psychology classes at school) and event-specific knowledge (e.g., when my psychology teacher told us about when she studied psychology at university). This organization of personal knowledge allows memories to be extracted and combined in many ways (Conway \& Playdell-Pearce 2000). The working self is an executive process that guides information encoding and retrieval depending on our current goals. For example, the goal in the Autobiographical Memory Test is to find a specific memory relevant to the cue word, the working self therefore interacts with the knowledge base to retrieve the required information.

A specific autobiographical memory can be retrieved through both directive and generative retrieval (Conway and Playdell Pearce 2000). Directive retrieval occurs when a cue in the environment immediately activates event specific knowledge. Generative retrieval requires an effortful 'top-down' process firstly activating broad information then general, which then activates event specific self-knowledge. According to the self-memory model (Conway and Playdell Pearce 2000), OGM occurs if the search is disrupted before activation is able to reach the bottom of the hierarchy.

Generative retrieval is when, in response to a request to retrieve a specific memory to a cue word, the individual forms a mental model of that request and then compares and evaluates memories activated during the against the model (Birch \& Davidson, 2007). This effortful process of retrieving a specific autobiographical memory is influenced by cognitive and motivational processes (Rawal \& Rice, 2012b). Higher cognitive (or executive) processes (e.g., working memory, inhibition) influence the likelihood that activated representations are consistent with the search criteria (e.g. a cue word "party"). Generative retrieval requires both the capacity to search for memory representations and to evaluate these against the memory model. Therefore, information needs to be held and updated in working memory and any irrelevant search information needs to be ignored (Conway \& Pleydell-Pearce 2000; William 
et al., 2007). The most comprehensive model to explain why this generative search process gets truncated at more general levels for people with depression is the CaR-FA-X model (Williams et al., 2007).

Williams et al., (2007) proposed that three mechanisms contribute to OGM by interfering with the search process. These are 'capture and rumination' $(\mathrm{CaR})$, 'functional avoidance' (FA), and reduced executive control (X). Capture and rumination $(\mathrm{CaR})$ refers to the idea that self-relevant information 'captures' cognitive resources and activates ruminative processing. Rumination involves focusing on abstract thoughts about the self in a repetitive way and is a common maladaptive emotion regulation strategy in depression (Schafer, Naumann, Holmes, Tuschen-Caffier \& Samson, 2017). Although often measured as one construct there are thought to be two sub-types of rumination (brooding rumination and pondering rumination) that differ in terms of adaptiveness. Treynor et al (2003) suggest that brooding is a maladaptive strategy associated with higher levels of depression, and that reflective pondering is a more adaptive, problem solving strategy. In relation to the CaR-FA$\mathrm{X}$ model, rumination uses cognitive resources and is, thus hypothesised to interfere with the ability to retrieve specific information about the past (Nolen-Hoeksema 2000; Treynor, Gonzalez \& Nolen-Hoeksema, 2003). Functional avoidance (FA) is a means of regulating negative affect by avoiding autobiographical content associated with distress. Executive control (X) refers to a broad range of cognitive strategies used to manage, focus on, plan and carry out tasks (Roberts, 1998). Executive function is a product of executive control (Funahashi and Andreau, 2013). Miyake et al, (2000) proposed a model of executive functioning comprising of three separate (but related) elements; i.e. inhibition, working memory and shifting. 'Inhibition' refers to the ability to deliberately suppress pre-potent (dominant) responses to achieve a more appropriate task-relevant response, 'updating and monitoring working memory representations' refers to the monitoring of incoming 
information while evaluating the relevance of information and discarding of old information (i.e. updating the current information stored in working memory), and 'shifting' relates to the switching between tasks (Mikaye et al., 2000) Deficits in executive control are hypothesised to interfere with the retrieval of specific autobiographical memories because of the need to hold information in mind (updating working memory) and ignore irrelevant information. Therefore if these skills are impaired the memory search is more likely to stop before retrieving the requested specific autobiographical information (Conway \& Pleydell-Pearce 2000; Williams et al., 2007).

Consistent evidence supports the CaR-FA-X model in adults with depression (Sumner et al., 2011). However, relatively few studies have examined these mechanisms in adolescents with depression and those that have produce mixed findings. There is evidence that Capture and Rumination is related to overgeneral memory in young people who have elevated depression symptoms. For example, experimentally induced rumination increased OGM in depressed adolescents but not in a non-depressed control group (Park, Goodyer and Teasdale 2004). Smets, Griffith, Wessel, Walschaerts \& Raes (2013) compared capture and rumination in young people with high and low levels of depression symptoms. Participants completed the AMT before and after a self-discrepancy induction. Following the experimental induction, rumination was significantly correlated with OGM only in the high depression group. Participants with elevated depression symptoms became more overgeneral in their memories after self-discrepant information captured their attention.

Functional avoidance (of a detailed memory) is hypothesized to emerge as a way of coping with negative emotions associated with exposure to a traumatic event. Memories are thought to be retrieved in a less specific manner to avoid the emotions triggered by recalling negatively charged autobiographical information (Williams, 2007). Therefore the memory search stops at the general level because this activates less negative emotion (Conway and 
Playdell Pearce 2000). Over time this emotion regulation strategy becomes habitual and occurs for all memories, whether positive or negative (Williams et al., 2007). In adolescents, Functional Avoidance is associated with overgeneral memory in those exposed to sexual abuse (Ogle et al., 2013), bereavement (Neshat Doost, Yule, Kalantari, Rohollah, Dyregrov \& Jobson, 2014) and family violence (Johnson, Greenhoot, Glisky \& McCloskey, 2005). Young people who had direct exposure to early trauma retrieved more overgeneral memories compared to young people who did not have exposure to early trauma (Hitchcock, Nixon and Weber 2014). Kuyken, Howell and Dalgleish (2006) found that OGM can also occur in the absence of trauma. They compared autobiographical memory retrieval between three groups, depressed adolescents with a history of trauma, depressed adolescents without a history of trauma, and never depressed adolescents. Depressed young people without a history of trauma had more OGM than either of the other groups, suggesting that exposure to trauma alone does not explain the development of OGM. Studies measuring functional avoidance in young people tend to infer avoidance by assessing exposure to early trauma as the measure of functional avoidance or by assessing general self-reported avoidance (Hitchcock et al, 2014). As far as we are aware, no previous study with adolescents has investigated the relationship between OGM and a more general cognitive avoidance strategy, although avoidance i.e. of internal psychological events, is a maladaptive emotion regulation strategy in young people with depression symptoms (Schafer, Naumann, Holmes, Tuschen-Caffier \& Samson, 2017). In the current study we assess functional avoidance as a cognitive avoidance strategy (Sumner, 2012) by evaluating the presence or absence of negative material recalled in response to the Autobiographical Memory Test.

Research on executive control (X) and OGM in adolescents with depression is inconclusive (Viglis, Silk \& Vance, 2015). Raes, Verstraeten, Bijttebier, Vasey and Dalgleish (2010) found that inhibitory control (a sub component of executive control) mediated the 
relationship between depression symptoms and OGM in a community group of 10 year olds. Inhibition was measured via a temperament questionnaire, but for a clearer understanding of the mechanism of impaired executive control studies need to include a behavioral inhibition task (Stewart, Hunter \& Rhodes, 2017), and to examine executive control in older adolescents. Additionally, conflicting evidence concerning executive control and OGM has been reported, Kuyken et al (2006) found no relationship between verbal fluency and OGM and Valentino et al., (2012) reported inhibition (via a colour word inference task) and OGM were not significantly correlated. Thus further work is needed to understand the relationship between executive control and OGM in young people.

There is some evidence that the components of the CaR-FA-X model may not operate in isolation from each other. Rawal and Rice found that young people at familial risk of developing depression who had high rumination and low executive control were more likely to exhibit OGM one year later (Rawal \& Rice 2012a). However, the measure of executive control used was generic and did not examine unique components of executive control that are thought to influence specific memory retrieval, including updating information in working memory and inhibiting irrelevant thoughts and memories (Sumner, 2012). Another recent study by Stewart, Hunter \& Rhodes (2018) examined the interaction between rumination and executive control in a group of healthy adolescents. Executive control was negatively associated with OGM but only in individuals with high levels of reflective pondering. No research in young people has yet looked at the relationship between OGM and the two rumination sub types separately in young people with depression symptoms. In a recent review of the relationship between exposure to trauma and OGM, (Stewart et al., 2017) reported that no study has yet included all components of the CaR-FA-X model with young people. 
The aim of this study therefore was to investigate overgeneral memory and to test the key mechanisms of the CaR-FA-X model (rumination, functional avoidance and executive functioning) in adolescents with elevated symptoms of depression. Multiple components of executive functioning (working memory, verbal fluency and inhibition) were also included. The components chosen for testing are directly related to the retrieval of overgeneral memory i.e. the ability to inhibit irrelevant thoughts and memories while monitoring and updating information relevant autobiographical knowledge while keeping task instructions in mind. Based on the CaR-FA-X model the study hypotheses are:

i) Adolescents with elevated symptoms of depression will report more overgeneral autobiographical memories than adolescents with minimal symptoms of depression

ii) Adolescents with elevated symptoms of depression will have significantly higher rumination, increased functional avoidance of negative affect, and impaired executive control compared to age and gender matched controls with low levels of depression.

\section{Method}

\section{Design}

This was a between-groups design with adolescents recruited from the community. The independent variable was level of depression symptoms ('elevated' or 'low' based on self-reported symptoms). The dependent variables were the number of overgeneral memories retrieved, rumination, functional avoidance, and three aspects of executive control - verbal fluency, inhibitory control (reaction time and errors), and working memory updating. A brief measure of IQ was used as a covariate. The sample size for each group was determined by a power analysis. Power was calculated using a planned analyses using the statistical 
programme $G *$ power. Based upon the mean between-group effect size of $d=0.86$ on overgeneral memory (i.e. Vrielynck. Delpus \& Philippot, 2007; Park et al. 2004), 28 participants were needed in each group.

\section{Participants}

Adolescents $(\mathrm{N}=58)$ aged 12-18 years were recruited from a pool of 215 participants who were pupils at a high school in England and who completed a measure of depressive symptoms (MFQ; Costello \& Angold 1995). Participants were recruited if they scored over 27 on the MFQ (5 male, 24 female) or below 12 on the MFQ (5 male, 24 female); The groups were matched on age, $t(56)-0.46, p=0.65$, gender $\chi^{2}(1)=0, p=1$ and ethnicity, $\chi^{2}(1)=$ 0.47, $\mathrm{p}=0.48$ (see Table 1$)$.

[Table 1 near here]

\section{Measures}

\section{Self-report questionnaires}

The Mood and Feelings Questionnaire (MFQ; Costello \& Angold, 1988). This was used to screen participants and determine eligibility for the study and group allocation. The MFQ is a 33 item self-report measure of depression in children and adolescents with good reliability and moderate validity (Burleson Daviss et al., 2006; Kent, Vostanins, \& Feehan, 1997; Wood, Kroll, Moore \&Harrington 1995). Each item is rated on a 3-point scale; 0 (not true) to 2 (true). Internal consistency was high (MFQ $\alpha=0.96$ ). A cut off of 27 and above was used to identify clinically significant levels of depression (Wood et al., 1995) and thus membership of the elevated group. MFQ scores of below 12 were used to identify a low depression group. This lower cut off was based on data from a clinical sample of 467 young people with a diagnosis of major depression (Goodyer et al., 2017) in which no participant with a diagnosis of major depression scored below 13 on the MFQ. Participants in the current 
study also completed the 13-item Brief Mood and Feelings Questionnaire (BMFQ: Angold et al., 1995) at the time of the research assessment (approximately 2 weeks later) to measure current symptoms of depression and confirm allocation to the low depression and high depression groups.

The Rumination Response Scale (RRS; Nolen-Hoeksema \& Morrow, 1991). This is a 22-item questionnaire asking participants what they habitually think, do or feel when they experience low mood. The measure has been validated for use with adolescents (Kuyken, Watkins, Holden, \& Cook, 2006). Internal consistency was high (RRS $\alpha=0.96)$. The RRS includes two 5 item sub-scales 'Brooding' and 'Reflective Pondering' that differ in relation to depression. (Treynor, Gonzalez, Nolen-Hoeksema 2003).

\section{Measures administered by the researcher}

The Autobiographical Memory Test (AMT; Williams \& Broadbent, 1986). This is a standardised test of autobiographical memory. Participants were asked to provide specific autobiographical memories in response to 10 emotional cue words (Loved, Excited, Relaxed, Lucky, Relieved, Angry, Failure, Hopeless, Lonely, Sad) that were presented in a random order.

Participants were told that the event they remembered could be important or trivial, recent or from a long time ago, but that the memory should be something that happened at a particular place and time. All participants were given an example before starting the test. They were told that if they were given the word 'party', it would be fine to say: 'I had a great time at Sara's party last Saturday' but that 'When I go to parties I have a good time' was not acceptable (because it did not specify a particular place and time). Two practice items were given (happy and sad) to make sure that participants understood the instructions.

The words were adapted from Heron et al., (2012) who carried out research on the psychometric properties of the test. They chose 5 positive and 5 negative words that were 
judged to be familiar to adolescents. The words were matched for familiarity using the University of Essex Children's Printed Word Database (Lovejoy, 2003). Positive words were; happy, excited, relaxed, lucky, relieved. Negative words were bored; failure, hopeless, lonely, sad. Heron et al., found that 'happy' and 'bored' showed poor discrimination in OGM. In this study 'happy' and 'bored'. were replaced with 'loved' and 'angry' as used by Kuyken and Dalgleish (2011).

Generated memories were tape-recorded and transcribed. Coding was based on the method used by Kuyken \& Dalgleish (2011). Two researchers, who were blind to participant's MFQ score, coded all the memories from each participant from transcripts. Specific memories were defined as events that lasted less than a day. Non-specific memories included extended memories (events that lasted a distinct, but prolonged period of time) and categoric memories (events that had occurred on multiple occasions with no defined time frame). Answers that did not refer to the past and non-responses were coded as 'not a memory'. Inter-rater reliability between two independent coders, who were blind to group membership of participants, was good $\kappa=0.78$. Discrepancies were discussed and an agreed code allocated.

\section{Assessment of Functional Avoidance}

To assess functional avoidance, memories generated in the autobiographical memory test were coded for positivity and negativity on a Likert scale ( -3 for very negative, 0 for neutral, and +3 for very positive). This measure of functional avoidance was based on the assumption that functional avoidance is a cognitive strategy used to regulate emotions (Sumner, 2012). Thus if participants did not recall specific negative or aversive details in response to prompts in the autobiographical memory task this would indicate functional avoidance (Schafer, Naumann, Holmes, Tuschen-Caffier \& Samson, 2017). Functional 
avoidance would therefore be indicated by recall of more positively valenced and fewer negatively valenced memories.

Inter-rater reliability between two independent coders, who were blind to group membership of participants, was high $\kappa=0.84$. There was no difference in the valence of memories recalled in response to positive and negative cue words so the mean valence across all 10 cue words was used as an index of functional avoidance. The Likert scale included both positive and negative numbers; therefore 7 was added to all scores to provide data suitable for statistical analysis.

\section{Assessment of IQ}

The Wechsler Abbreviated Scale of Intelligence (WASI II; Wechsler, 2011). The WASI II is a brief, well-standardised assessment of intellectual abilities for 6 to 89 -year-olds. Two subtests (Vocabulary and Matrix Reasoning) were used to provide an estimate of fullscale intelligence quotient (FSIQ). In the vocabulary subtest, participants were required to define a word that was presented to them orally. The Matrix Reasoning consisted of 30 incomplete matrices presented in a stimulus book. Participants were asked to look at each incomplete matrix and choose one item from a selection of five figures at the bottom of each page to correctly complete the matrix. The WASI has strong psychometric properties, including good test- retest reliability and internal and concurrent validity (Garland, 2005; McCrimmon \& Smith, 2013).

\section{Executive function tasks}

The Controlled Oral Word Association Test (COWAT; Benton \& Hamsher, 1976). This was used to measure verbal fluency. Participants were asked to list as many words as possible in 60 seconds that begin with the letters $\mathrm{F}, \mathrm{A}$ and $\mathrm{S}$. The task requires the ability to organise verbal information, to initiate retrieval, and maintain a search set in working memory and inhibit inappropriate responses (Swan \& Carmelli, 2002). Verbal fluency is a 
standard measure of neuropsychological assessment, with good levels of test-re-test reliability and validity (Harrison, Buxton, Hussain \& Wise, 2000).

The Hayling Sentence Completion task (HSC; Burgess \& Shallice, 1996). This is a measure of inhibition that detects difficulties in suppressing pre-potent responses. The task involves asking participants to listen to incomplete sentences read by the researcher (e.g., "London is a very big - ") and then provide a word that completes the sentence either appropriately (e.g., 'city'; Part A) or inappropriately (e.g., 'banana'; Part B). The task has moderate validity and reliability (Strauss et al., 2006) and has been used with adolescents (Robinson, Goddard, Dritschel, Wisley, \& Howlin, 2009).

Inhibition in this test is measured in two ways - the number of errors and reaction time. Errors include word endings that finish the sentences appropriately or are associated with the meaning of the sentence. To analyse both measures, raw scores were converted into scaled scores, 8 indicating "good performance" and 1 indicating signs of cognitive impairment (Burgess \& Shallice 1996).

The Keep track task (KTT; originally adapted by Miyake et al., (2000) from Yntema (1963)). This is a reliable and valid measure of updating working memory (Thompson \& Gathercole, 2006; Conway et al., 2005). Participants were shown a list of six target categories (e.g. animals, furniture, fruit) and exemplars (dog, table, apple), compiled using an updated and extended version of Battig and Montague (1969) norms (Overschelde, Rawson, \& Dunlosky, 2004). They were asked to familiarise themselves with the words and make sure they know which exemplar belongs to each category. At the beginning of each trial a single tone was played and several target categories were displayed on the lower half of the computer screen. Fifteen words, including 2 or 3 exemplars from each of six possible categories were then presented serially in a pseudo-randomized order for 2 seconds per word. Target categories remained on the screen during the trial. The task was to recall the last word 
presented in each of the target categories. Thus participants have to continuously update their working memory for the target categories. After each trial, participants were asked to recall the exemplars used in that trial.

Before the task, participants practised on two trials with three target categories. The task itself consisted of four trials with three target categories, four trials with four target categories and one trial with five target categories. The total number of words presented was 33 and the percentage of words recalled correctly was the measure of interest.

\section{Procedure}

Two hundred and fifteen students completed the MFQ and rumination questionnaire in their classroom and indicated that they were willing to take part in further research (126 students from an original screening number of 341 did not want to be involved in future research). Seventy two students who scored 12 or below, or 27 or above on the MFQ were invited to take part in the research of whom 58 agreed to take part. The interval between screening and testing was approximately 1 week.

Individual meetings were arranged with each participant in a quiet room at school, during the school day. Administration of the tasks took between 45-55 minutes. Participants completed the brief MFQ first. The cognitive tests were then administered in a standardised order; Verbal Fluency, Hayling Sentence Completion Task, Keep Track task, Autobiographical Memory task and the WASI II. All adolescents who completed the research tasks were entered into a prize draw to win a $£ 10$ Amazon voucher.

\section{Ethics}

The University of Reading Research Ethics Committee provided ethical approval for this study. Written informed consent was obtained from all participants over 16 years, and parental informed consent and adolescent assent were obtained for all participants under 16 years. 
Information about the study was given to young people and their parents. All students who agreed to be contacted, met study criteria, and had parental consent (or were over 16 years and consented for themselves) were invited to take part. Before testing began, the study was explained to each adolescent by the researcher. As agreed with the school and with young people and their parents, and in line with the school safeguarding procedures, young people who reported elevated symptoms of depression were identified to a nominated member of pastoral care.

\section{Results}

\section{Preliminary Analyses and Analytic Plan}

Continuous data were screened in relation to the assumptions of parametric tests (Tabachnick \& Fidell, 2007). Where assumptions were violated, confirmatory analyses were conducted by running analyses with 1000 bootstrap samples. All results were consistent, suggesting that the original analyses were robust to the violations of assumptions, so the results based on the original (non-bootstrapped) analyses are presented for simplicity.

To confirm that young people with elevated depression symptoms at screening were still reporting similar symptom levels at the next research session, depression scores on the Brief MFQ were examined. All participants had levels of depression that were consistent with their screening MFQ scores. Group differences in OGM were examined using an independent samples t-test. Next, group differences on each mechanism of the CaR-FA-X model were examined using multivariate (MANCOVA) analyses of variance, controlling for multiple tests. Group (low depression vs elevated depression) was the independent variable, and executive function, rumination and functional avoidance measures were dependent variables. Because IQ is associated with executive control (Mahone et al., 2002; Arffa 2007), the WASI II IQ score was used as a covariate to ensure any deficits could be attributed to executive control difficulties. 


\section{Between group differences in symptoms of depression and IQ}

Mean levels of depression symptoms in the elevated group were significantly higher than in the control group (see Table 1). The mean MFQ score in the elevated groups was similar to that reported in a clinical sample of depressed adolescents (Goodyer et al., 2017). The mean IQ score in both groups was average (Shaw et al., 2006).

[Table 2 near here]

\section{Hypothesis testing}

The first hypothesis was that adolescents with elevated symptoms of depression would retrieve more overgeneral autobiographical memories than adolescents with low levels of depression. There was a significant between-groups difference in the number of overgeneral memories retrieved, $t(56)=1.37, p<.001, \mathrm{~d}=1.1$. As expected, adolescents with elevated depression symptoms retrieved more overgeneral memories than those with low levels of depression (see Table 2).

Descriptive statistics for all measures assessing components of the CaR-FA-X model are presented in Table 2. The CaR-FA-X model was tested in a MANCOVA with group as the independent variable, functional avoidance, rumination and three variables measuring executive control as dependent variables and WASI II as covariate. There was a significant multivariate effect of group $F(6,450)=7.63, p<.001$ showing that the depressed and the non-depressed groups significantly differed on the CaR-FA-X components. This was followed up with univariate $\mathrm{F}$ tests to examine each element of the CaR-FA-X model separately. There was a significant effect of group on brooding rumination, $F(1,55)=7.32$, $p<.001$ and reflective pondering rumination $F(1,55)=7.32, p<.001$; both types of rumination were higher in the group with elevated depression. There was no significant effect of group on functional avoidance, $F(1,55)=3.41, p=.07)$. In response to the cue words, adolescents in the elevated depression group did not recall more positive or fewer negative 
memories than those in the low depression group. Participants in both groups provided a wide range of positive and negative memories in response to both positive and negative cue words. There was a significant univariate effect of group on verbal fluency $F(1,55)=7.32, p=$ .009 ; the elevated depression group produced significantly fewer words than the low depression group. There was also a significant group difference in working memory $F(1,55)$ $=11.90, p=.001$; adolescents with elevated symptoms of depression had poorer working memory than the low depressed group. Contrary to the hypothesis there was no significant difference in inhibition between groups, either for the number of errors made $F(1,55)=$ $.450, p=.51$, or for reaction time, $F(1,55)=3.86, p=.06$.

\section{Discussion}

The aim of this study was to test if the CaR-FA-X model of overgeneral autobiographical memory is applicable to adolescents with high symptoms of depression. As predicted, young people with elevated depression symptoms recalled significantly fewer specific memories than the low depression group. The CaR-FA-X model was partly supported. Young people who reported higher symptoms of depression also reported significantly more rumination and had impaired executive control, in terms of verbal fluency and working memory. However, they had no deficits in inhibition and there was no evidence of functional avoidance; i.e. they did not report fewer negative or more positive autobiographical memories than young people without depression symptoms.

To investigate the executive control component of the CaR-FA-X model, we included a broad measure of executive control, verbal fluency, and two sub processes of executive control thought to be involved in the retrieval of a memory search, updating working memory and inhibition (Conway and Playdell-Pearce 2000).This selection of different executive components reflects the notion that separate executive function components may have different influences on the retrieval search of autobiographical memories (Rawal and Rice 
$2012 b$ ). For example, to retrieve a specific memory it is necessary to inhibit irrelevant memories that come to mind and keep in mind instructions and the aim of the search, thus working memory must be used and updated (Williams et al., 2007). As hypothesised, adolescents who had high depression symptoms had poorer verbal fluency and were less able to update working memory than young people with low depression. Contrary to the hypothesis, the elevated depression group did not differ from the low depression group in their ability to inhibit a verbal response, suggesting that inhibitory control did not interfere with the memory search. This is not consistent with Raes et al., (2010) who found that self reported inhibition mediated the relationship between depression symptoms and OGM. However, in the current study, both groups performed well on the measure of inhibition and there was a possible ceiling effect. When participants were asked to give a word that did not make sense at the end of a sentence both groups used similar, successful strategies to help inhibit their initial responses i.e. such as scanning the room for random items.

Inhibition is not a unitary construct (Nigg, 2000) and can be split into behavioural inhibition (the ability to resist acting impulsively) and cognitive inhibition (the inhibition of prepotent mental representations including thoughts and memories). Here we found no group difference on a behavioural inhibition task and previously Valentino et al., (2012) found no association between cognitive inhibition and OGM (on the colour word inference task. However, Raes and colleagues (2010) did report a significant association between overgeneral memory and inhibition as assessed on a self-report questionnaire. Therefore, the relationship between inhibition and OGM in depressed adolescents needs to be investigated further with a more sensitive measure that capture all elements of the construct.

Common symptoms of depression in adolescents include a lack of motivation, concentration and increased fatigue (Goodyer et al, 2017, Orchard, 2016). These more general symptoms of depression are a plausible explanation for deficits in cognitive tasks, 
including autobiographical memory recall, and executive functioning tasks, which typically require effort and concentration. However, in this study there were no between group difference in IQ, suggesting that deficits in executive control and autobiographical memory specificity were not explained by a lack of motivation, poor concentration, or fatigue.

There was also support for the rumination mechanism of the CaR-FA-X model. Ruminating (i.e. repetitive and circular thinking about one's problems) uses cognitive resources that are believed to compete with the resources needed to retrieve specific memories. This finding is in line with previous research (Park, Goodyer, Teasdale, 2004) supporting the rumination mechanism within the CaR-FA-X model. There are two types of rumination; 'brooding' and 'reflective pondering'. In our sample adolescents with elevated symptoms of depression engaged more in both forms of rumination compared to healthy controls. Previous research has suggested an interaction between executive control and rumination i.e. when adolescents with familial risk of developing depression had lower executive control and ruminated more they recalled less specific memories on the AMT (Rawal and Rice, 2012b). We were not able to test the relationship between rumination and executive control due to limited sample size. However, the group differences on rumination and executive control suggests that future work looking at the relationship between these two mechanisms in adolescents with low mood would be worthwhile.

There was no evidence that functional avoidance (i.e. avoiding negative memory content as a cognitive strategy to decrease exposure to negative feelings) was higher in adolescents with elevated depression symptoms. Functional avoidance was measured by coding the valence of the memories retrieved on the Autobiographical Memory Task; if depressed young people were avoiding negative autobiographical content as a way of managing negative affect, we would expect to see less negative content recalled. There was no difference between the groups in terms of memory valence. Therefore we did not find 
support for the hypothesis that adolescents with high depression symptoms were functionally avoiding negative material to dampen negative affect. This method used to assess functional avoidance in this study is a new and untested way to assess functional avoidance. Rating the emotional valance of autobiographical memories has good face validity but ratings made by participants of their own memories would improve face validity further.

We did not directly measure participants' experience of trauma; to do so in a community sample would present considerable ethical challenges. It is possible that functional avoidance is a specific mechanism only associated with experience of severe trauma - and this is best examined in a clinical setting where appropriate therapeutic support is available if required. We also did not measure for lifetime exposure to negative or positive experiences, which might plausibly differ between the elevated and low risk groups. Specifically, because of increased exposure to negative life events (including possible trauma) young people in the elevated depression group may have had a larger mental 'catalogue' of negative experiences to draw on, and thus their retrieval of memories that did not differ in affective tone from the non-depressed group may reflect a positively biased retrieval).

This study is the first to evaluate the three components/mechanisms of the CaR-FA-X model with adolescents with elevated depression symptoms, and to measure separate executive functions likely to be involved in the impaired executive control mechanism and the two sub-components of rumination. Participants were recruited from a non-clinical population of young people aged 13 to 18 years old so were not help seeking or diagnosed with depression. However, they were assessed using a well-established self-report measure of depression and the groups were clearly distinct in relation to symptom severity but matched well on other salient variables. The elevated depression group reported very high depression symptoms, similar in severity to levels reported in clinical samples (e.g. Orchard et al, 2016), 
therefore the current results are likely to generalise to other adolescents with high levels of depression symptoms. Well-established measures of rumination, autobiographical memory, and elements of executive control were used. Although a well-established measure was used to assess inhibition, it is important to note recent concerns about the sensitivity of the task, (e.g. the Hayling Sentence Completion Test) that were developed for use with neuropsychological groups, to detect potentially less marked deficits in clinical disorders (Synder et al., 2015). Therefore, possible deficits with inhibitory control should be explored further using more specific and sensitive tests of inhibition e.g., the Stop Signal task (Verbruggen et al, 2008).

The results of this study make a significant contribution to understanding the mechanisms of OGM to adolescents. This is of practical as well as theoretical interest because this developmental period marks the onset of most first episodes of depression (Costello, Erkanli \& Angold, 2006) as well as significant cognitive development, including the maturation of the prefrontal cortex (Paus, Keshavan, Giedd, 2008; Steinberg 2005). Therefore understanding key psychological mechanisms associated with depression, such as executive control and rumination, is particularly important because they may also inform the development of new interventions to prevent or treat depression. If impaired cognitive processes and impaired autobiographical memory constitute a vulnerability for the development of depression early identification of these difficulties and targeted interventions may prevent future onset of depression. Likewise, because cognitive processes such as concentration, attention and memory are so critical to educational and occupational functioning and are clearly impaired during episodes of depression these outcomes should be assessed before and after treatment to ensure that adolescents with depression are fully recovered. If cognitive processes do not improve after routine treatment for depression it may 
be necessary to develop additional or adjunctive treatments to target these areas alongside interventions designed to improve emotional symptoms of depression.

\section{References}

Angold, A., Costello, E. J., Messer, S. C., Pickles, A., Winder, F., \& Silver, D. (1995) The development of a short questionnaire for use in epidemiological studies of depression in children and adolescents. International Journal of Methods in Psychiatric Research, 5, 237 249.

Arffa, S. (2007). The relationship of intelligence to executive function and nonexecutive function measures in a sample of average, above average, and gifted youth. Archives of Clinical Neuropsychology, 22 (8), 969 - 978.

Battig, W. F., \& Montague, W. E. (1969). Category norms of verbal items in 56 categories A replication and extension of the Connecticut category norms. Journal of experimental Psychology, 80(3p2), 1.

Benton, A. L., \& Hamsher, K. D. S.(1976). Multilingual aphasia examination. Iowa City: University of Iowa.

Birch, L. S., \& Davidson, K. M. (2007). Specificity of autobiographical memory in depressed older adults and its relationship with working memory and IQ. British Journal of Clinical Psychology, 46(2), 175-186.

Boden, J. M., Fergusson, D. M., \& Horwood, L. J. (2007). Anxiety disorders and suicidal behaviours in adolescence and young adulthood: findings from a longitudinal study. Psychological medicine, 37(3), 431-440.

Brittlebank, A .D., Scott, J., Williams, J. M. G., \& Ferrier, I. N.(1993). Autobiographical memory in depression: State or trait marker? British Journal of Psychiatry, 162 , 118 - 121.

Burgess, P. W., \& Shallice, T. (1996). Response suppression, initiation and strategy use following frontal lobe lesions. Neuropsychologia, 34(4), 263-272.

Burleson Daviss, W., Birmaher, B., Melhem, N. A., Axelson, D. A., Michaels, S. M., \& Brent, D. A. (2006). Criterion validity of the Mood and Feelings Questionnaire for depressive episodes in clinic and non- clinic subjects. Journal of Child Psychology and Psychiatry, 47(9), 927-934.

Conway, M. A., \& Pleydell - Pearce, C. W. (2000). The construction of autobiographical memories in the self - memory system. Psychological Review, 107 (2), 261 - 288. 
Conway, A. R., Kane, M. J., Bunting, M. F., Hambrick, D. Z., Wilhelm, O., \& Engle, R. W. (2005). Working memory span tasks: A methodological review and user's guide. Psychonomic bulletin \& review, 12(5), 769-786.

Costello, E. J., \& Angold, A. (1988). Scales to assess child and adolescent depression checklists, screens and nets. Journal of the American Academy of Child and Adolescent Psychiatry, 27(6), 726-737.

Costello, J. E., Erkanli, A., \& Angold, A. (2006). Is there an epidemic of child or adolescent depression? Journal of Child Psychology and Psychiatry 47 (12), 1263 - 1271.

Dalgleish, T., \& Werner-Seidler, A. (2014). Disruptions in autobiographical memory processing in depression and the emergence of memory therapeutics. Trends in cognitive sciences, 18(11), 596-604.

Dalgleish, T., Williams, J. M. G., Golden, A. J., Perkins, N., Barrett, L. F., Barnard, P., ...Watkins, E. (2007). Reduced specificity of autobiographical memory and depression: The role of executive control. Journal of Experimental Psychology: General, 136 (1), 23 - 42

Fergusson, D. M., Horwood, L. J., Ridder, E. M., \& Beautrais, A. L. (2005). Subthreshold depression in adolescence and mental health outcomes in adulthood. Archives of General Psychiatry, 62 (1), 66 - 72.

Funahashi, S., \& Andreau, J. M. (2013). Prefrontal cortex and neural mechanisms of executive function. Journal of Physiology-Paris, 107(6), 471-482.

Garland, T. (2005). The Wechsler Abbreviated Scale of Intelligence (WASI): An overview and case studies in occupational assessment. Journal of Occupational Psychology, Employment and Disability, 7(2), 125-129.

Goodyer, I. M., Reynolds, S., Barrett, B., Byford, S., Dubicka, B., Hill, J., ... \& Senior, R. (2017). Cognitive-behavioural therapy and short-term psychoanalytic psychotherapy versus brief psychosocial intervention in adolescents with unipolar major depression (IMPACT): a multicentre, pragmatic, observer-blind, randomised controlled trial. Health Technology Assessment (Winchester, England), 21(12), 1.

Green, H., McGinnity, Á., Meltzer, H., Ford, T., \& Goodman, R. (2005). Mental health of children and young people in Great Britain, 2004.

Groeben, M., Perren, S., Stadelmann, S., \& von Klitzing, K. (2011). Emotional symptoms from kindergarten to middle childhood: associations with self-and other-oriented social skills. European child \& adolescent psychiatry, 20(1), 3-15.

Harrison, J. E., Buxton, P., Husain, M., \& Wise, R. (2000). Short test of semantic and phonological fluency: Normal performance, validity and test- retest reliability. British Journal of Clinical Psychology, 39(2), 181-191. 
Heron, J., Crane, C., Gunnell, D., Lewis, G., Evans, J., \& Williams, J. M. G. (2012). 40,000 memories in young teenagers: Psychometric properties of the Autobiographical Memory Test in a UK cohort study. Memory, 20(3), 300-320.

Hitchcock, C., Nixon, R. D. V., \& Weber, N. (2014). A review of overgeneral memory in child psychopathology. British Journal of Clinical Psychology, 53 (2), 170 - 193.

Hermans, D., Vandromme, H., Debeer, E., Raes, F.,Demyttenaere, K., Brunfaut, E., et al. (2008).Overgeneral autobiographical memory predicts diagnostic status in depression. Behaviour Research and Therapy, 46, 668-677.

Johnson, R. J., Greenhoot, A. F., Glisky, E., \& McCloskey, L. A. (2005). The relations among abuse, depression, and adolescents' autobiographical memory. Journal of Clinical Child and Adolescent Psychology, 34(2), 235-247.

Kent, L., Vostanis, P., \& Feehan, C. (1997). Detection of major and minor depression in children and adolescents: evaluation of the Mood and Feelings Questionnaire. Journal of Child Psychology and Psychiatry, 38(5), 565-573.

Kuyken, W., \& Dalgleish, T. (2011). Overgeneral autobiographical memory in adolescents at risk for depression. Memory, 19 (3), $241-250$.

Kuyken, W., Howell, R., \& Dalgleish, T. (2006). Overgeneral autobiographical memory in depressed adolescents with, versus without, a reported history of trauma. Journal of Abnormal Psychology, 115 (3), 387 - 396.

Kuyken, W., Watkins, E., Holden, E., \& Cook, W. (2006). Rumination in adolescents at risk for depression. Journal of Affective Disorders, 96 (1 - 2), 39 - 47.

Lewinsohn, P. M., Rohde, P., Klein, D. N., \& Seeley, J. R. (1999). Natural course of adolescent major depressive disorder: I. Continuity into young adulthood. Journal of the American Academy of Child \& Adolescent Psychiatry, 38(1), 56-63.

Mahone, E. M., Hagelthorn, K. M., Cutting, L. E., Schuerholz, L. J., Pelletier, S. F., Rawlins, C., ... \& Denckla, M. B. (2002). Effects of IQ on executive function measures in children with ADHD. Child Neuropsychology, 8(1), 52-65.

McCrimmon, A. W., \& Smith, A. D. (2013). Review of the Wechsler Abbreviated Scale of Intelligence, (WASI-II).

Miyake, A., Friedman, N. P., Emerson, M. J., Witzki, A. H., Howerter, A., \& Wager, T. D. (2000). The unity and diversity of executive functions and their contributions to complex "frontal lobe" tasks: A latent variable analysis. Cognitive psychology, 41(1), 49-100.

Neshat Doost, H. T., Yule, W., Kalantari, M., Rezvani, S. R., Dyregrov, A., \& Jobson, L. (2014). Reduced autobiographical memory specificity in bereaved Afghan adolescents. Memory, 22(6), 700-709. 
Nigg, J. T. (2000). On inhibition/disinhibition in developmental psychopathology: views from cognitive and personality psychology and a working inhibition taxonomy. Psychological bulletin, 126(2), 220.

Nolen-Hoeksema, S. (2000). The role of rumination in depressive disorders and mixed anxiety/depressive symptoms. Journal of abnormal psychology, 109(3), 504.

Nolen - Hoeksema, S. (1991). Responses to depression and their effects on the duration of depressive episodes. Journal of Abnormal Psychology, 100 (4), 569 - 582.

Ogle, C. M., Block, S. D., Harris, L. S., Goodman, G. S., Pineda, A., Timmer, S., ... \& Saywitz, K. J. (2013). Autobiographical memory specificity in child sexual abuse victims. Development and psychopathology, 25(2), 321-332.

Orchard, F., Pass, L., Marshall, T., \& Reynolds, S. (2017). Clinical characteristics of adolescents referred for treatment of depressive disorders. Child and Adolescent Mental Health, 22(2), 61-68.

Park, RJ, Goodyer, I and Teasdale, J. (2002). Categoric overgeneral autobiographical memory in adolescents with major depressive disorder. Psychological Medicine, 32: 267276.

Park, R. J., Goodyer, I. M., \& Teasdale, J. D. (2004). Effects of induced rumination and distraction on mood and overgeneral autobiographical memory in adolescent Major Depressive Disorder and controls.Journal of Child Psychology and Psychiatry, 45, 996-1006.

Paus, T., Keshavan, M., \& Giedd, J. N. (2008). Why do many psychiatric disorders emerge during adolescence?. Nature Reviews Neuroscience, 9(12), 947-957.

Raes, F., Hermans, D., de Decker, A., Eelen, P., \& Williams, J. M. G. (2003). Autobiographical memory specificity and affect regulation: an experimental approach. Emotion, 3(2), 201.

Raes, F., Verstraeten, K., Bijttebier, P., Vasey, M. W., \& Dalgleish, T. (2010). Inhibitory control mediates the relationship between depressed mood and overgeneral memory rec all in children. Journal of Clinical Child and Adolescent Psychology, 39 (2), 276 - 281.

Rawal, A., \& Rice, F. (2012a). Examining overgeneral autobiographical memory as a risk factor for adolescent depression. Journal of the American Academy of Child \& Adolescent Psychiatry, 51 (5), 518 - 527. doi: 10.1016/j.jaac.2012.02.025

Rawal, A., \& Rice, F. (2012b). A longitudinal study of processes predicting the specificity of autobiographical memory in the adolescent offspring of depressed parents. Memory, 20 (5), 518 - 526. DOI: 10.1080/09658211.2 012.683011

Roberts, A. C., Robbins, T. W., \& Weiskrantz, L. E. (1998). The prefrontal cortex: Executive and cognitive functions. Oxford University Press. 
Robinson, S., Goddard, L., Dritschel, B., Wisley, M., \& Howlin, P. (2009). Executive functions in children with autism spectrum disorders. Brain and cognition, 71(3), 362-368.

Schäfer, J. Ö., Naumann, E., Holmes, E. A., Tuschen-Caffier, B., \& Samson, A. C. (2017). Emotion regulation strategies in depressive and anxiety symptoms in youth: A meta-analytic review. Journal of youth and adolescence, 46(2), 261-276.

Shaw, P., Greenstein, D., Lerch, J., Clasen, L., Lenroot, R., Gogtay, N. E. E. A., ... \& Giedd, J. (2006). Intellectual ability and cortical development in children and adolescents. Nature, 440(7084), 676-679.

Smets, J., Griffith, J. W., Wessel, I., Walschaerts, D., \& Raes, F. (2013). Depressive symptoms moderate the effects of a self-discrepancy induction on overgeneral autobiographical memory. Memory, 21(6), 751-761.

St Clair-Thompson, H. L., \& Gathercole, S. E. (2006). Executive functions and achievements in school: Shifting, updating, inhibition, and working memory. The Quarterly Journal of Experimental Psychology, 59(4), 745-759.

Steinberg, L. (2005). Cognitive and affective development in adolescence. Trends in cognitive sciences, 9(2), 69-74

Strauss, Esther, Sherman, Elizabeth Spreen \& Otfried (2006). A Compendium of Neuropsychological Tests: Administration, Norms, and Commentary. Oxford: Oxford University Press.

Stewart, T. M., Hunter, S. C., \& Rhodes, S. M. (2017). A narrative synthesis of the applicability of the $\mathrm{CaR}-\mathrm{FA}-\mathrm{X}$ model in child and adolescent populations: a systematic review. Memory, 1-30.

Stewart, T. M., Hunter, S. C., \& Rhodes, S. M. (2018). A prospective investigation of rumination and executive control in predicting overgeneral autobiographical memory in adolescence. Memory \& cognition, 1-15.

Sumner, J. A. (2012). The mechanisms underlying overgeneral autobiographical memory: An evaluative review of evidence for the Ca R - FA - X model. Clinical Psychology Review, 31 (1), 34 - 48.

Sumner, J. A., Griffith, J. W., \& Mineka, S. (2011). Examining the mechanisms of overgeneral autobiographical memory: capture and rumination, and impaired executive cont rol. Memory, 19 (2), 169 - 183.

Snyder, H. R., Miyake, A., \& Hankin, B. L. (2015). Advancing understanding of executive function impairments and psychopathology: bridging the gap between clinical and cognitive approaches. Frontiers in psychology, 6.

Swales, M. A., Williams, J. M. G., \& Wood, P. (2001). Specificity of autobiographical memory and mood disturbance in adolescents. Cognition \& Emotion, 15(3), 321-331. 
Swan, G. E., \& Carmelli, D. (2002). Evidence for genetic mediation of executive control: A study of aging male twins. Journal of Gerontology: Psychological Sciences, 57B, 133-143.

Tabachnick, B. G., \& Fidell, L. S. (2007). Multivariate analysis of variance and covariance. Using multivariate statistics, 3, 402-407.

Treynor, W., Gonzalez, R., \& Nolen - Hoeksema, S. (2003). Rumination reconsidered: A psychometric analysis. Cognitive Therapy and Research, 27 (3), 247 - 259.

Valentino, K., Bridgett, D. J., Hayden, L. C., \& Nuttall, A. K. (2012). Abuse, depressive symptoms, executive functioning, and overgeneral memory among a psychiatric sample of children and adolescents. Journal of Clinical Child \& Adolescent Psychology, 41(4), 491498.

Van Overschelde, J. P., Rawson, K. A., \& Dunlosky, J. (2004). Category norms: An updated and expanded version of the norms. Journal of Memory and Language, 50(3), 289-335.

Verboom, C. E., Sijtsema, J. J., Verhulst, F. C., Penninx, B. W., \& Ormel, J. (2014). Longitudinal associations between depressive problems, academic performance, and social functioning in adolescent boys and girls. Developmental psychology, 50(1), 247.

Verbruggen, F., \& Logan, G. D. (2008). Response inhibition in the stop-signal paradigm. Trends in cognitive sciences, 12(11), 418-424.

Vilgis, V., Silk, T. J., \& Vance, A. (2015). Executive function and attention in children and adolescents with depressive disorders: a systematic review. European child \& adolescent psychiatry, 24(4), 365-384.

Vrielynck, N., Delpus, S., \& Philippot, P. (2007). Overgeneral autobiographical memory and depressive disorder in children. Journal of Clinical and Adolescent Psychology, 36, 95-105.

Wechsler, D. (2011). WASI-II: Wechsler abbreviated scale of intelligence--. Psychological Corporation.

Williams, J. M. G., Barnhofe r, T., Crane, C., Hermans, D., Raes, F., Watkins, E., \& Dalgleish, T. (2007). Autobiographical memory specificity and emotional disorder. Psychological Bulletin, 133 (1), 122 - 148.

Williams, J. M. G., \& Broadbent, K. (1986). Autobiographical memory in suicide attempters. Journal of Abnormal Psychology, 95 (2), 144 - 149.

Wood, A., Kroll, L., Moore, A., \& Harrington, R. (1995). Properties of the Mood and Feelings Questionnaire in adolescent psychiatric outpatients: A research note. Journal of Child Psychology and Psychiatry, 36, 327-334.

Yntema, D. B. (1963). Keeping track of several things at once. Human factors, 5(1), 7-17. 
